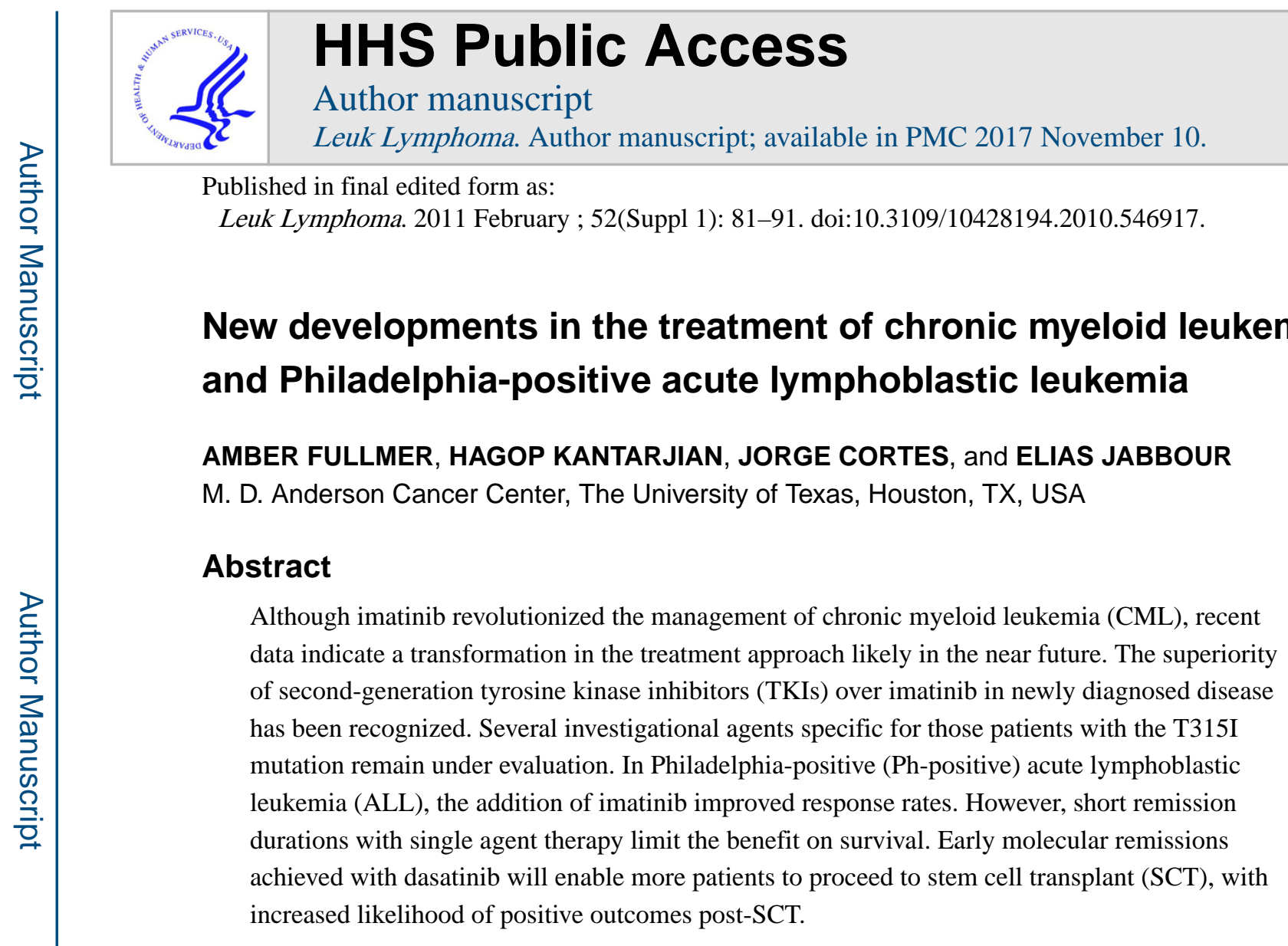

\title{
Keywords
}

Lymphoid leukemia; myeloid leukemias and dysplasias; chemotherapeutic approaches

\section{Chronic myeloid leukemia}

The most remarkable advancement in chronic myeloid leukemia (CML) was the development of imatinib, a tyrosine kinase inhibitor (TKI) that inhibits BCR-ABL. Imatinib was proved superior to the previous standard of care regimen in the International Randomized Study of Interferon and STI571 (IRIS). Patients with chronic phase CML (CML-CP) were randomized to receive treatment with imatinib $400 \mathrm{mg}$ daily $(n=553)$ or interferon- $a$ in combination with low dose cytarabine $(n=553)$ [1]. Treatment with imatinib resulted in higher rates of hematologic and cytogenetic responses, less progression to advanced forms of CML (accelerated phase [CML-AP] and blast phase [CML-BP]), and improved tolerability. The most recent report of the IRIS trial confirms sustained treatment responses and durable remissions through 8 years of follow-up, with a low likelihood of disease progression. Imatinib is currently approved for newly diagnosed patients with CMLCP, CML-AP, CML-BP, CML-CP after failure of interferon- $a$ therapy, and Philadelphiapositive acute lymphoblastic leukemia (Ph-positive ALL) [2].

Correspondence: Elias Jabbour, MD, Department of Leukemia, The University of Texas, M. D. Anderson Cancer Center, 1515 Holcombe Blvd., Box 428, Houston, TX 77030, USA. Tel: 713-792-4764. Fax: 713-794-4297. ejabbour@ mdanderson.org. 


\section{Imatinib resistance}

Despite the results with imatinib, some patients will have resistance or intolerance to the drug. Approximately $33 \%$ of patients are refractory to treatment with imatinib [3]. Primary resistance, defined as failure to achieve response, has been classified as primary hematologic resistance, reported in $2-4 \%$ of cases, and primary cytogenetic resistance, reported in 15$25 \%$ of cases [4]. Primary resistance has not been attributed to mutations in BCR-ABL, but associations have been suggested with increased transcript levels of prostaglandinendoperoxide synthase 1/cyclooxgenase 1 (PTGS/COX1). Thus, elevated levels of this gene may identify patients with primary resistance to imatinib [5,6]. Secondary resistance is described as achievement of response followed by loss of the hematologic or cytogenetic response. Over half of the resistance displayed following treatment with imatinib occurs as a result of mutations in the BCR-ABL kinase domain [7-9]. Further treatment can be tailored to the mutation that is detected. For example, the T315I mutation, reported to occur in 4$15 \%$ of imatinib resistance, confers additional resistance to dasatinib, nilotinib, and bosutinib [10,11]. In the case that this mutation becomes present, further treatment should include clinical trials with investigational agents or hematopoietic stem cell transplant (HSCT). However, not all BCR-ABL point mutations confer resistance to the available TKIs. Decreased sensitivity to nilotinib is apparent for mutants Y253H, E255K/V, and F359C/V, whereas mutants E255K/V, V299L, and F317L are insensitive to dasatinib [12]. Additional treatment should incorporate the TKI that retains sensitivity to the particular mutation. In some cases, the above mutations are not present, and therapy should be guided by patient comorbidities as well as adverse effects associated with the drug.

Baseline mutational assessment for newly diagnosed patients is currently not recommended. The identification of mutations has not correlated with sensitivity of imatinib or response $[13,14]$. However, in the case of suboptimal response or failure of a particular therapy, mutational analysis is recommended $[15,16]$.

\section{High dose imatinib}

The dose of imatinib in newly diagnosed patients with CML-CP is $400 \mathrm{mg}$ daily. In the case of suboptimal response or failure, imatinib dose escalation is likely to be successful in patients who had previously achieved a cytogenetic response and have not developed any mutations conferring resistance to imatinib. Some mutations display sensitivity with increased concentrations of imatinib [17-19]. In addition, dose escalation can potentially overcome resistance resulting from subtherapeutic levels of imatinib [15,20]. In the IRIS trial, dose escalation to $600 \mathrm{mg}$ or $800 \mathrm{mg}$ daily was permitted for failure to achieve complete hematological response (CHR) at 3 months or at least a minor cytogenetic response (CyR) by 12 months, loss of a major cytogenetic response (MCyR) at any time, disease progression including increase in white blood cell count, development of CML-AP or CML-BP, and loss of CHR or MCyR. Thirty-nine patients randomized to the imatinib arm of the study had an imatinib dose escalation due to the above mentioned criteria [21]. After 12 months of treatment with the escalated dose per the IRIS protocol, approximately half of the patients had positive results. Of those patients without CHR at 3 months $(n=7)$, six (86\%) demonstrated CHR at 12 months post-dose-escalation. Two of the eight patients without at least a minor CyR achieved a MCyR within 12 months, and MCyR was observed 
in an additional two patients within 24 months of treatment. For those patients with loss of MCyR ( $n=18)$, nine had reached MCyR after 12.5 months of treatment with escalated imatinib dosing. Complete CyR (CCyR) occurred in three patients by 30 months of treatment. Forty-eight patients had dose escalation of imatinib according to the European LeukemiaNet (ELN) recommendations [15]. Improvements were reported as follows: CHR was achieved in 6/7 patients with escalated dose, 6/11 patients with failure achieved MCyR after 12 months of increased-dose imatinib, and CCyR was achieved in 2/10 patients with previous failure at 18 months. Three years following dose escalation per either IRIS or ELN criteria, the estimated progression-free survival (PFS) was $89 \%$ and overall survival (OS) was $84 \%$.

In a separate evaluation of patients with CML-CP who developed hematologic or cytogenetic failure, dose escalation proved effective for patients with a previous cytogenetic response. Eighty-four patients failing imatinib had dose escalation from $400 \mathrm{mg}$ daily to 800 $\mathrm{mg}$ daily, or from $300 \mathrm{mg}$ daily to $600 \mathrm{mg}$ daily in those patients with prior dose reduction due to toxicity [22]. Cytogenetic response was achieved in 47 patients (75\%) with previous cytogenetic failure, of whom $34(40 \%)$ attained CCyR. In contrast, three patients with hematologic failure had a cytogenetic response, with CCyR achieved in only one patient $(5 \%)$. Of those patients who had never achieved a cytogenetic response, rates of 2- and 3year event-free survival (EFS), transformation-free survival (TFS), and OS were significantly lower than in patients with previous cytogenetic response. While myelosuppression requiring dose reduction was apparent, the majority of patients received the full escalated dose throughout 12 months of therapy.

Though imatinib $400 \mathrm{mg}$ daily is the approved Food and Drug Administration (FDA) dose for CML-CP, several investigations have suggested that initial treatment with a higher dose may improve response. In CML-AP and CML-BP, escalated dosing improves response rates, time to progression, and overall survival [23]. In the Rationale and Insight for Gleevec HighDose Therapy (RIGHT) trial, 115 patients with newly diagnosed CML-CP treated with imatinib $400 \mathrm{mg}$ twice daily attained high rates and early occurrence of molecular and cytogenetic responses [24]. Major molecular response (MMR) increased over time, with $48 \%$ in MMR at 6 months, $54 \%$ at 12 months, and $63 \%$ at 18 months, with a median time to MMR of 8.3 months. MCyR was $90 \%$ at 12 months and $96 \%$ at 18 months, with CCyR $85 \%$ at 12 months and $83 \%$ at 18 months. In comparison with previous investigations of standard dose imatinib, patients treated according to the RIGHT trial experienced higher and faster rates of molecular and cytogenetic responses. However, a randomized evaluation of imatinib $400 \mathrm{mg}$ daily versus $800 \mathrm{mg}$ daily in 476 newly diagnosed patients with CML-CP did not show a difference in MMR at 12 months [25]. Treatment discontinuation was similar in patients treated with imatinib $400 \mathrm{mg}$ daily (15.9\%) and imatinib $800 \mathrm{mg}$ daily (19.7\%). At 24 months, high dose imatinib was not associated with any significant improvement in MMR, CCyR, EFS, PFS, or OS as compared to standard dose imatinib in the TKI optimization and selectivity study (TOPS) [26]. In both treatment arms, patients who experienced one or less interruption during imatinib therapy achieved higher rates and shorter time to MMR and increased rates of CCyR. Dose interruptions longer than 5 days occurred in $71 \%$ of patients treated with high dose imatinib and $44 \%$ of patients who received the standard dose. The occurrence of adverse events including grade 3-4 
neutropenia, thrombocytopenia, rash, diarrhea, and myalgia was more frequent with imatinib $800 \mathrm{mg}$ daily compared with imatinib $400 \mathrm{mg}$ daily. To improve outcomes, interruption of therapy should be avoided, and dose intensity should be maintained throughout treatment. In the standard dose arm, median dose intensity was $400 \mathrm{mg}$, while in the high dose arm, median dose intensity was $728 \mathrm{mg}$. Of patients in the high dose arm who maintained a dose of 600-800 mg daily versus those who received less than $600 \mathrm{mg}$ daily, significant improvements were noted in achievements of MMR at 12 months (62.4\% vs. 34.1\%), MMR at 18 months (75.2\% vs. $40.3 \%)$, time to and duration of MMR, and CCyR at 12 months ( $89.6 \%$ vs. $70.3 \%$ ) in patients continuing on a higher daily dose. Though $800 \mathrm{mg}$ daily appeared tolerable, frequent dose interruptions limit the ability to maintain dose intensity, and therefore $400 \mathrm{mg}$ daily remains the recommended dose for initial treatment of CML-CP. Furthermore, $800 \mathrm{mg}$ daily has been reported in some studies to shorten the time to MMR and CCyR when compared to imatinib $400 \mathrm{mg}$ daily with or without the addition of interferon; however, longer duration of follow-up is necessary to determine a correlation with survival [27].

\section{Dasatinib}

Dasatinib is an orally available TKI approved for treatment of all phases of CML with resistance or intolerance to imatinib and Ph-positive ALL [28]. Dasatinib has multiple advantages over imatinib and can overcome several mechanisms of resistance, proving beneficial in patients who have secondary resistance to imatinib [29]. For example, dasatinib binds to BCR-ABL in both the open and closed conformation, whereas imatinib binds only in the closed conformation [30]. For dasatinib this results in an increased affinity to BCR$\mathrm{ABL}$, and it has a potency that is approximately 325 -fold greater than that of imatinib [31,32]. In addition, dasatinib also has the ability to inhibit src activity, which blocks BCRABL activity through a separate mechanism.

The efficacy of dasatinib following imatinib failure has been reported. In the Src/ABL tyrosine kinase inhibition activity research trials of dasatinib (START)-C, dasatinib $70 \mathrm{mg}$ twice daily demonstrated efficacy in 387 patients with CML-CP, resistant or intolerant to imatinib [33,34]. At 24 months' follow-up, MCyR was achieved in $62 \%$ of patients, with CCyR in 53\% of patients [35]. PFS was $80 \%$ and OS $94 \%$. Neutropenia and thrombocytopenia were reported to be of grade $3-4$ in $50 \%$ and $49 \%$ of patients, respectively. Notable non-hematologic grade 3-4 adverse effects included pleural effusion (9\%), dyspnea (6\%), bleeding (4\%), diarrhea (3\%), and fatigue (3\%).

START-R further evaluated dasatinib in comparison with imatinib $800 \mathrm{mg}$ daily in patients with CML-CP who had failed therapy with imatinib 400-600 mg daily [36]. Patients were randomized to receive therapy with dasatinib $70 \mathrm{mg}$ twice daily ( $n=101)$ or imatinib $800 \mathrm{mg}$ daily $(n=49)$. Greater cytogenetic responses were achieved with dasatinib, as reported rates of MCyR were 53\% for dasatinib and 33\% for imatinib, and CCyR rates were $40 \%$ for dasatinib and $16 \%$ for imatinib. MMR was also significantly improved with dasatinib (16\%) compared with imatinib (4\%). In addition, dasatinib appeared better tolerated, as significantly fewer patients discontinued therapy with dasatinib versus imatinib. Pleural 
effusions were common in the dasatinib group (17\%); however, only $3 \%$ were reported as grade 3 , and none were reported as grade 4 .

Though initial investigations studied dasatinib dosed twice daily, once-daily dosing has proved to be as effective, with better tolerability [37-40]. Four dasatinib dosing regimens were evaluated in CML-CP: $100 \mathrm{mg}$ daily ( $n=167), 50 \mathrm{mg}$ twice daily ( $n=168), 140 \mathrm{mg}$ daily ( $n=167)$, and $70 \mathrm{mg}$ twice daily ( $n=168)$. After 24 months of follow-up, dasatinib 100 $\mathrm{mg}$ daily demonstrated MCyR and CCyR rates similar to the other investigated dosing schemes [38]. Estimates for PFS were reported as follows: $80 \%$ for $100 \mathrm{mg}$ daily, $76 \%$ for $50 \mathrm{mg}$ twice daily, $75 \%$ for $140 \mathrm{mg}$ daily, and $76 \%$ for $70 \mathrm{mg}$ twice daily. Overall survival estimates were $91 \%$ for dasatinib $100 \mathrm{mg}$ daily, $90 \%$ for $50 \mathrm{mg}$ twice daily, $94 \%$ for $140 \mathrm{mg}$ daily, and $88 \%$ for $70 \mathrm{mg}$ twice daily. Reported PFS and OS at 36-months of follow-up continue to favor $100 \mathrm{mg}$ daily as the optimal dose [39]. Dasatinib $100 \mathrm{mg}$ daily was associated with significantly fewer pleural effusions of any grade (14\%) in comparison to the other dasatinib treatment regimens (23-25\%). Of those reported, only $2 \%$ of pleural effusions in the $100 \mathrm{mg}$ daily group were classified as grade 3-4 versus $4-5 \%$ in the other investigated regimens. In a separate dose optimization study of dasatinib in CML-AP and CML-BP, $140 \mathrm{mg}$ daily displayed comparable response rates and lower rates of adverse effects, particularly pleural effusions, compared with $70 \mathrm{mg}$ twice daily [40]. As a result of these studies, $100 \mathrm{mg}$ daily has been approved for patients with CML-CP and $140 \mathrm{mg}$ daily for patients with advanced phase disease [28].

In comparison with standard dose imatinib, dasatinib $100 \mathrm{mg}$ daily in CML-CP was associated with significantly higher and faster rates of CCyR and MMR [41]. Patients with newly diagnosed CML were randomized to receive dasatinib $100 \mathrm{mg}$ daily $(n=259)$ or imatinib $400 \mathrm{mg}$ daily $(n=260)$ in the Dasatinib versus Imatinib Study in Treatment-Naive CML Patients (DASISION) (Table I). At 12 months, CCyR was significantly higher among patients treated with dasatinib versus imatinib $(77 \%$ vs. $66 \%, p=0.007)$ as well as the rate of MMR ( $46 \%$ vs. $28 \%, p=0.002$ ). Shorter time to achievement of CCyR was evident with dasatinib. After 3, 6, and 9 months of treatment with dasatinib, the rates of CCyR were 54\%, $73 \%$, and $78 \%$, while rates of CCyR with imatinib were only $31 \%, 59 \%$, and $67 \%$ at the same time points. Time to achievement of MMR was also reduced with dasatinib. The rates of MMR achieved after 3, 6, and 9 months of dasatinib were $8 \%, 27 \%$, and $39 \%$ versus $0.4 \%, 8 \%$, and $18 \%$ with imatinib. Progression to more advanced forms of CML occurred less frequently in patients receiving dasatinib (1.9\%) versus imatinib (3.5\%). Both drugs were well tolerated, and adverse events were similar between groups. Pleural effusion was only reported for patients taking dasatinib (10\%), with all events reported as grade $1(2 \%)$ or grade $2(8 \%)$. Drug discontinuation was infrequent among both groups, with $84 \%$ of patients receiving dasatinib and $81 \%$ of patients receiving imatinib throughout the duration of study. Achieving CCyR and MMR quickly is associated with more favorable long-term outcomes. The ability of dasatinib to rapidly induce high rates of response suggests the potential role of this agent in newly diagnosed patients. 


\section{Nilotinib}

Nilotinib, similar to imatinib, binds to the inactive conformation of BCR-ABL and has no ability to inhibit scr activity, but is a more potent and selective inhibitor [42-44]. It is approved for the treatment of patients with newly diagnosed CML-CP, (before CML-CP) CML-CP and CML-AP who are resistant or intolerant to imatinib [45]. At 24-months of follow-up, nilotinib $400 \mathrm{mg}$ twice daily administered to 321 patients with CML-CP led to MMR in 28\% and CCyR in $46 \%$ of those treated [46]. MCyR and CCyR were maintained for $77 \%$ and $84 \%$ of patients at 24 months. Of note, patients with complete hematologic response at initiation of nilotinib had more favorable responses and outcomes than those patients who had lost their hematologic response. Common grade 3-4 laboratory abnormalities and adverse effects included elevated lipase (17\%), hypophosphatemia (16\%), hyperglycemia (12\%), elevated bilirubin $(8 \%)$, neutropenia (31\%), thrombocytopenia $(31 \%)$, and anemia (10\%). In 137 heavily pretreated patients with CML-AP, nilotinib led to durable responses and favorable outcomes [47]. The rate of MCyR was 32\%, with CCyR achieved in $20 \%$ of patients. At 24 months, the estimated OS was $67 \%$.

Nilotinib has displayed favorable outcomes in the front-line setting (Table I). Patients at the M. D. Anderson Cancer Center with newly diagnosed CML-CP received treatment with nilotinib $400 \mathrm{mg}$ twice daily ( $n=51$ ) [48]. Within 6 months of therapy, almost all patients reached CCyR (98\%) and 76\% achieved a MMR. In a separate phase II evaluation of newly diagnosed patients with CML-CP, nilotinib led $96 \%$ of patients to achieve CCyR and $85 \%$ reached MMR within 1 year of therapy [49]. Nilotinib $300 \mathrm{mg}$ twice daily also produced high rates of CCyR and MMR in a study conducted by the All-Ireland Cooperative Oncology Research Group [50]. The high response rates and favorable toxicity profile associated with nilotinib warrant further investigation of front-line therapy for CML-CP. In the phase III randomized trial of nilotinib 300 or $400 \mathrm{mg}$ twice daily versus imatinib $400 \mathrm{mg}$ daily in newly diagnosed patients with CML-CP (EN-ESTnd; Evaluating Nilotinib Efficacy in Clinical Trials of Newly Diagnosed Ph+CML Patients), both regimens of nilotinib were associated with higher rates of MMR and CCyR at 12 months [51]. Patients were randomized to nilotinib $300 \mathrm{mg}$ twice daily ( $n=282$ ), nilotinib $400 \mathrm{mg}$ twice daily ( $n=281$ ), or imatinib $400 \mathrm{mg}$ daily ( $n=283$ ). At 12 months, MMR and CCyR were significantly higher for those patients treated with nilotinib. The MMR was $44 \%$ for nilotinib $300 \mathrm{mg}$, $43 \%$ for nilotinib $400 \mathrm{mg}$, and $22 \%$ for imatinib ( $p<0.001$ ). Time to achievement of MMR was shorter for both nilotinib $300 \mathrm{mg}$ ( 8.6 months) and nilotinib $400 \mathrm{mg}$ (11 months) compared with imatinib (median not yet achieved). CCyR reported for nilotinib $300 \mathrm{mg}$, nilotinib 400 $\mathrm{mg}$, and imatinib was $80 \%, 78 \%$, and $65 \%$, respectively $(p<0.001)$. In addition, rate of progression to advanced disease was lower for those patients receiving nilotinib $300 \mathrm{mg}$ and nilotinib $400 \mathrm{mg}$ versus imatinib ( $<1 \%$ and $<1 \%$ vs. $4 \%$ ). Dose intensity was maintained for each treatment group with the median dose reported as $592 \mathrm{mg}$ daily for nilotinib $300 \mathrm{mg}$ twice daily, $779 \mathrm{mg}$ for nilotinib $400 \mathrm{mg}$ twice daily, and $400 \mathrm{mg}$ daily for imatinib. Rates of adverse events necessitating drug discontinuation were comparable between all study arms (5\% nilotinib $300 \mathrm{mg}, 9 \%$ nilotinib $400 \mathrm{mg}$, 7\% imatinib). There were no occurrences of QT interval above $500 \mathrm{~ms}$ in either the nilotinib group or the imatinib group. In a subset analysis of Japanese patients ( $n=79)$, the rate of MMR at 12 months was twice as high with nilotinib $300 \mathrm{mg}$ and nilotinib $400 \mathrm{mg}$ versus imatinib (57\% and 50\% vs. 24\%) [52]. CCyR rates 
were similar between groups and were as follows: $77 \%$ for nilotinib $300 \mathrm{mg}, 83 \%$ for nilotinib $400 \mathrm{mg}$, and $76 \%$ for imatinib. Though long-term follow up is lacking in the frontline setting, the superiority of nilotinib versus imatinib led to the FDA approval of nilotinib in newly diagnosed patients and suggests a modification of current treatment algorithms likely in the near future [53].

\section{Investigational agents}

Several agents with novel mechanisms are under evaluation for CML. Bosutinib (SKI-606), a dual Src/ABL inhibitor, has displayed activity in Ph-positive leukemias [54,55]. In a phase I/II study of 299 patients with CML-CP resistant or intolerant to imatinib, 58\% of evaluable patients achieved a MCyR, with 46\% reported as CCyR [55]. Grade 3-4 adverse effects and laboratory abnormalities included rash (9\%), diarrhea (8\%), hypermagnesemia (11\%), and increased alanine transaminase (ALT) (10\%). Grade 3-4 hematologic toxicity, including thrombocytopenia (23\%), neutropenia (14\%), and anemia (9\%), was common. While bosutinib maintains activity in patients with various BCR-ABL mutations, it is not active against the T315I mutation.

Other investigational agents that do display activity against the T315I mutation include omacetaxine, AP24534, PHA-739358, and DCC-2036. Omacetaxine, semi-synthetic homoharringtonine available for subcutaneous administration, is a multitargeted protein synthase inhibitor that works through a mechanism independent of BCR-ABL inhibition and displays activity regardless of mutational status [56-58]. In adult patients with CML-CP, CML-AP, or CML-BP, subcutaneous omacetaxine was administered at $1.25 \mathrm{mg} / \mathrm{m}^{2}$ twice daily for 14 days every 28 days until hematologic response, followed by maintenance dosing with $1.25 \mathrm{mg} / \mathrm{m}^{2}$ twice daily for 7 days every 28 days [59]. Of 90 patients enrolled, analysis was possible for 66. All patients had failed prior therapy with imatinib and had the T315I mutation, and $79 \%$ had failed therapy with two or more TKIs. In CML-CP, $27.5 \%$ of patients had a cytogenetic response, of which $15 \%$ were MCyR sustained for 6 or more months, and 15\% achieved MMR. Activity was maintained in advanced disease with one patient achieving CCyR. Myelosuppression was the most frequent adverse effect leading to treatment delays in 50\% of patients. A separate analysis of two long-term phase II studies in CML confirmed the safety of omacetaxine [60].

AP24534 is an orally available multikinase inhibitor. In a phase I evaluation of 42 patients with CML, the maximum tolerated dose of $60 \mathrm{mg}$ daily has been reached [61]. Of the evaluable patients with CML, MCyR has been achieved in 48\%, and CCyR in 33\%. Of the seven patients with T315I CML-CP evaluable for response, CCyR was reported for 57\%. Eight patients with T315I CML-AP or CML-BP were evaluable, of whom 13\% had CCyR. Commonly reported toxicities included nausea (20\%), fatigue (15\%), vomiting (15\%), headache (13\%), arthralgia (10\%), hot flush (10\%), increased glucose (10\%), increased lipase (10\%), muscle spasms (10\%), and rash (10\%). At the $60 \mathrm{mg}$ dose, grade 3-4 elevations of amylase and lipase occurred in one-third of patients, with two patients experiencing pancreatitis.

Danusertib hydrochloride (PHA-739358) is an aurora kinase inhibitor currently under phase I evaluation in CML and Ph-positive ALL. Administration via intravenous infusion over $3 \mathrm{~h}$ 
daily for 7 consecutive days represents the current dosing regimen under study [62]. Doses ranging from $90 \mathrm{mg} / \mathrm{m}^{2}$ to $200 \mathrm{mg} / \mathrm{m}^{2}$ have been tested; however, the maximum tolerated dose has not yet been established. Of 15 patients with documented T315I mutations, six have reported responses including cytogenetic, hematologic, and clinical improvement.

Another agent still in phase I studies is DCC-2036, which belongs to a new class called switch pocket inhibitors that bind to five structural pockets involved in the endogenous 'switch' mechanism used by the ABL kinase to conformationally control the state of activity [63]. DCC-2036 is selective for ABL, FLT3, and Src family kinases.

\section{Philadelphia-positive acute lymphoblastic leukemia}

Ph-positive ALL occurs in 20-30\% of patients [64,65]. Long-term disease-free survival remains poor for patients treated with traditional chemotherapy; however, the addition of TKIs to the therapy of Ph-positive ALL has improved outcomes in this subset of leukemia. Allogeneic SCT (aSCT) represents the only curative option, and current recommendations support eligible patients to proceed to aSCT in first complete remission (CR). Single agent therapy with imatinib was first reported to induce high response rates; however, remission durations were short, likely due to mutations in the BCR-ABL kinase domain. Dasatinib has the ability to overcome several limitations with imatinib, and recent data have supported the use of this agent with or without intensive chemotherapy. Early molecular responses with dasatinib reduce the disease burden, allowing more patients to proceed to aSCT and lengthen survival.

\section{Imatinib}

The first evaluations with imatinib monotherapy focused on relapsed or refractory elderly patients, likely not eligible for future aSCT. In CML-BP and relapsed and refractory ALL, single agent imatinib displayed activity at doses ranging from 300 to $1000 \mathrm{mg}$ per day [66]. Response rates of $70 \%$ were promising; however, only $20 \%$ of patients achieved CR. In 48 patients with relapsed Ph-positive ALL, imatinib 400-600 mg daily led $27 \%$ of patients to sustained hematologic response lasting 4 or more weeks in duration. Despite this, the median time to progression was 2.2 months, and overall survival was 4.9 months [67].

Data have suggested a benefit in response rates compared with intensive chemotherapy in newly diagnosed elderly patients [68]. In patients aged 55 years or older with de novo $\mathrm{Ph}$ positive ALL or CML-BP, imatinib $600 \mathrm{mg}$ daily $(n=27)$ produced superior responses compared with multiagent chemotherapy $(n=26)$. CR for patients receiving imatinib was 96\%; $85 \%$ achieved CR, and $11 \%$ attained CR without peripheral blood count recovery. In the multiagent chemotherapy arm, CR was only achieved in $50 \%$ of patients, with the majority represented as $\mathrm{CR}$ without peripheral blood count recovery. In elderly patients, imatinib $800 \mathrm{mg}$ daily in combination with prednisone led to CR in all patients evaluable for response ( $n=29$ ) [69]. However, fewer than half of the patients evaluated were alive and in CR after 10 months ( $n=13)$. While single agent imatinib, or in combination with steroids, represents a feasible option for elderly patients to achieve $\mathrm{CR}$, short durations of remission will unlikely lead to any improvements in survival. 
In younger patients fit to tolerate intensive chemotherapy, imatinib in combination with chemotherapy produces high quality CR enabling eventual aSCT (Table II). Both concurrent (administration at the same time) and sequential administration (alternating administration) of imatinib are practical approaches. In early investigations, the M. D. Anderson Cancer Center included imatinib $400 \mathrm{mg}$ daily for 14 days concurrently with HCVAD (hyperfractionated cyclophosphamide, vincristine, doxorubicin, and dexamethasone) as induction and consolidation therapy [70]. Imatinib was also incorporated into maintenance therapy with vincristine and prednisone at a continuous dose of $600 \mathrm{mg}$ daily. Modifications to the initial regimen dosed imatinib at $600 \mathrm{mg}$ daily for 14 days during course one of induction, $600 \mathrm{mg}$ daily administered continuously through consolidation, and $800 \mathrm{mg}$ daily administered continuously through maintenance, followed by indefinite imatinib therapy [71]. In 54 patients with imatinib naive, de novo, or minimally treated Ph-positive ALL, 93\% achieved CR. Of 47 patients with de novo disease, 14 proceeded to aSCT in first CR. Three-year OS was significantly improved in patients aged 40 years or less who proceeded to aSCT (90\%) compared with those who did not (33\%). In comparison with HCVAD, the addition of imatinib improved 3-year remission durations and survival.

The Japan Adult Leukemia Study Group (JALSG) ALL202 Ph-positive arm alternated imatinib $600 \mathrm{mg}$ daily in combination with methotrexate and cytarabine with single agent imatinib in 24 newly diagnosed patients during remission induction therapy [72]. CR was achieved in $96 \%$ of patients, with $63 \%$ eventually proceeding to aSCT. In a separate study conducted by the Group for Research on Adult Acute Lymphoblastic Leukemia (GRAALL), chemotherapy alternated with imatinib and steroids produced $72 \%$ CR in 30 newly diagnosed patients [73]. In this evaluation, the addition of imatinib improved OS and relapse-free survival when compared with similar patients who did not receive imatinib.

Though the incorporation of a TKI improved response rates, the exact point in treatment to include imatinib as well as the duration of therapy to maximize outcomes required further clarification. Different designs include imatinib during initial induction therapy, while others have reported benefit with initiation during consolidation treatment. In the German Multicenter Acute Lymphoblastic Leukemia trial, two separate cohorts were evaluated to determine which administration schedule of imatinib more greatly reduced $\mathrm{BCR}-\mathrm{ABL}$ transcript levels [74]. The first cohort consisted of imatinib alternating with chemotherapy, while the second cohort administered imatinib concurrently with chemotherapy. Though the reduction in BCR-ABL transcripts did not correlate with survival outcomes, the transcript level became undetectable in more patients in the concurrent arm (52\%) compared with the sequential arm (19\%). In the GRAAPH-2003 study, good early responders, defined by corticosensitivity and chemosensitivity, were enrolled in the study during the first course of consolidation therapy, which consisted of mitoxantrone, cytarabine, intrathecal chemotherapy, and continuous imatinib $600 \mathrm{mg}$ daily [75]. Those patients who did not display early corticosensitivity and chemosensitivity were administered induction consisting of imatinib $800 \mathrm{mg}$ daily with vincristine and dexamethasone (DIV). For all patients enrolled ( $n=45$ ), the CR rate was reported as $96 \%$, indicating the benefit of imatinib at varying doses administered during induction or consolidation. The high $\mathrm{CR}$ rate achieved with imatinib in this study allowed all patients with a donor to proceed to aSCT ( $n=22$ ). Imatinib $800 \mathrm{mg}$ daily in combination with HCVAD was most recently compared to an 
imatinib-based regimen including the TKI in combination with vincristine and dexamethasone [76]. At the time of reporting, 83 of the 118 newly diagnosed patients enrolled were evaluable. After two courses of induction/consolidation, a $100 \% \mathrm{CR}$ rate was reported for the imatinib-based treatment group $(n=42)$ versus $95 \%$ for imatinib-HCVAD $(n=41)$. No significant difference between imatinib-based and imatinib-with-HCVAD treatments was apparent in survival at 2 years.

Imatinib therapy in Ph-positive ALL is limited by primary and secondary resistance. Approximately $25-30 \%$ of patients display primary resistance and secondary resistance occurs rapidly, with an estimated time to development of 2 months after treatment initiation $[67,77]$. The majority of mutations in the kinase domain in Ph-positive ALL are recognized after treatment with a TKI [78]. In patients not treated with imatinib, mutations were not detected at the time of relapse; however, $88 \%$ of patients who were treated with TKIs displayed BCR-ABL mutations at the time of relapse [79]. While these ideas require investigation, intermittent TKI therapy, dual TKI therapy, and combinations of TKIs with agents that suppress clonal evolution have been proposed to minimize resistance and eventual relapse. Despite historical data reporting disappointing responses with immunologic therapy in Ph-positive ALL, imatinib in combination with interferon- $a$ displayed encouraging outcomes in a small series of patients [80].

Central nervous system (CNS) relapse will develop readily in the absence of prophylactic interventions $[81,82]$. TKIs alone offer limited prophylaxis for CNS disease [83,84]. Considering the lack of penetration into the CNS with TKIs, intrathecal chemotherapy should routinely be incorporated to reduce the likelihood of relapse in the CNS.

\section{Dasatinib}

Initial studies with dasatinib were in relapsed and refractory patients. The START-L trial included 36 heavily pretreated patients with Ph-positive ALL or CML-BP [85]. All patients had previously failed treatment with imatinib, and had persistent disease despite aSCT (42\%), chemotherapy (89\%), and interferon- $a$ (8\%). Dasatinib $70 \mathrm{mg}$ twice daily enabled rapid disease control with major hematologic response achieved at a median of 1.8 months. CCyR was achieved by $58 \%$ of patients at 8 months of treatment. In the Gruppo Italiano Malattie Ematologiche dell' Adulto (GIMEMA) LAL 1205 protocol, dasatinib $70 \mathrm{mg}$ twice daily was combined with intrathecal chemotherapy in 48 patients, 34 of whom were evaluable for response [86]. All patients achieved CHR. Dose optimization studies of dasatinib in CML concluded that once-daily therapy was as effective, with fewer adverse effects, than twice-daily dosing [37-40]. Patients with Ph-positive ALL resistant or intolerant to imatinib were randomized to dasatinib $140 \mathrm{mg}$ daily $(n=40)$ or $70 \mathrm{mg}$ twice daily $(n=44)$ [87]. Though not statistically significant, major hematologic response and MCyR were higher in the once-daily (38\% and 70\%) versus the twice-daily (32\% and 52\%) group. In addition, PFS and OS were longer in the once-daily (3 and 9.1 months) versus the twice-daily (4 and 6.5 months) group. Toxicities between groups were similar, though the occurrence of pleural effusion was lower in the once-daily (18\%) versus the twice-daily (32\%) treatment group. These results confirm that dasatinib $140 \mathrm{mg}$ once daily rapidly 
induces remissions in heavily pretreated patients with Ph-positive ALL, and serves as the currently approved dose [28].

Several studies have evaluated dasatinib in combination with chemotherapy [88-90]. Dasatinib $140 \mathrm{mg}$ daily in combination with vincristine and dexamethasone was evaluated in 22 newly diagnosed patients over 55 years of age [88]. Dasatinib was incorporated into consolidation and maintenance therapy. The complete hematologic response was $95 \%$; however, doses reductions for patients over 70 years of age have been recommended due to a high rate of adverse events, including four deaths. At the M. D. Anderson Cancer Center, dasatinib in combination with the HCVAD regimen has been investigated in newly diagnosed $(n=39)$ as well as relapsed and refractory patients $(n=23)$ [89,90]. Dasatinib 100 $\mathrm{mg}$ daily was included for 14 days with each cycle of induction and consolidation, with continuous dasatinib administered during maintenance. In newly diagnosed patients, CR was reported for $95 \%$ of patients and CCyR was achieved in $79 \%$ of patients [89]. Complete molecular remission was reported in 56\% and MMR in $21 \%$. In relapsed and refractory patients with Ph-positive ALL or CML-BP, 65\% achieved CR and 26\% had CR without complete platelet recovery [90]. MCyR was achieved in $90 \%$ and MMR in 65\% of patients. The addition of dasatinib to intensive chemotherapy provides sufficient control of leukemia to enable eligible patients to proceed to SCT. With dose reductions, intensive regimens in combination with dasatinib can be administered to elderly patients to improve response rates and potentially improve survival.

\section{Conclusion}

While imatinib drastically changed the management of CML, advanced-generation TKIs have the potential to transform the current approach to treatment. Resistance and intolerance to imatinib require alternative therapies. High-dose imatinib does not consistently demonstrate a benefit in newly diagnosed patients; however, in patients with previous cytogenetic response and lack of mutations, this strategy can overcome resistance due to subtherapeutic levels of the drug. A substantial amount of data confirm the safety and efficacy of dasatinib and nilotinib after imatinib failure. In newly diagnosed patients with CML, dasatinib and nilotinib demonstrate similar rates of response, and shorten the time to achievement of clinical milestones. Data for front-line therapy with these agents require additional follow-up before conclusive outcomes on survival can be assessed. Currently available TKIs provide no benefit for patients who harbor the T315I mutation; however, investigational therapies such as omacetaxine and AP24534 display encouraging results in this subset of patients.

Although Ph-positive ALL has traditionally been associated with a poor prognosis, the introduction of TKIs has led to improved response rates. High quality CR rates and early molecular responses achieved with TKIs increase the eligibility for SCT and likelihood of positive outcomes. Monotherapy should be reserved for patients unable to tolerate intensive chemotherapy. Dose reductions of multiagent chemotherapy for elderly patients with adequate performance status should further be explored. The management of $\mathrm{Ph}$-positive ALL with advanced-generation TKIs as well as investigational agents will eventually lead to durable remissions and lengthened survival. 


\section{References}

1. O'Brien SG, Guilhot F, Larson RA, et al. Imatinib compared with interferon and low-dose cytarabine for newly diagnosed chronic-phase chronic myeloid leukemia. N Engl J Med. 2003; 348:994-1004. [PubMed: 12637609]

2. Gleevec (imatinib) [package insert]. East Hanover, NJ: Novartis Pharmaceuticals Corp; Feb. 2010

3. Hochhaus A, O'Brien SG, Guilhot F, et al. Six-year follow-up of patients receiving imatinib for the first-line treatment of chronic myeloid leukemia. Leukemia. 2009; 23:1054-1061. [PubMed: 19282833]

4. Shah NP. Medical management of CML. Hematology Am Soc Hematol Educ Program. 2007:371375. [PubMed: 18024653]

5. Deininger MW. Optimizing therapy of chronic myeloid leukemia. Exp Hematol. 2007; 35(Suppl 1): 144-154. [PubMed: 17379100]

6. Zhang WW, Cortes JE, Yao H, et al. Predictors of primary imatinib resistance in chronic myelogenous leukemia are distinct from those in secondary imatinib resistance. J Clin Oncol. 2009; 27:3642-3649. [PubMed: 19506164]

7. Soverini S, Colarossi S, Gnani A, et al. Contribution of ABL kinase domain mutations to imatinib resistance in different subsets of Philadelphia-positive patients: by the GIMEMA Working Party on Chronic Myeloid Leukemia. Clin Cancer Res. 2006; 12:7374-7379. [PubMed: 17189410]

8. Shah NP, Nicoll JM, Nager B, et al. Multiple BCR-ABL kinase domain mutations confer polyclonal resistance to the tyrosine kinase inhibitor imatinib (STI571) in chronic phase and blast crisis chronic myeloid leukemia. Cancer Cell. 2002; 2:117-125. [PubMed: 12204532]

9. Ernst T, Erben P, Muller MC, et al. Dynamics of BCR-ABL mutated clones prior to hematologic or cytogenetic resistance to imatinib. Haematologica. 2008; 93:186-192. [PubMed: 18223278]

10. Jabbour E, Kantarjian H, Jones D, et al. Characteristics and outcomes of patients with chronic myeloid leukemia and T315I mutation following failure of imatinib mesylate therapy. Blood. 2008; 112:53-55. [PubMed: 18403620]

11. Modugno M, Casale E, Soncini C, et al. Crystal structure of the T315I Abl mutant in complex with the aurora kinases inhibitor PHA-739358. Cancer Res. 2007; 67:7987-7990. [PubMed: 17804707]

12. Muller MC, Cortes JE, Kim DW, et al. Dasatinib treatment of chronic-phase chronic myeloid leukemia: analysis of responses according to preexisting BCR-ABL mutations. Blood. 2009; 114:4944-4953. [PubMed: 19779040]

13. Willis SG, Lange T, Demehri S, et al. High-sensitivity detection of BCR-ABL kinase domain mutation in imatinibnaïve patients: correlation with clonal cytogenetic evolution but not response to therapy. Blood. 2005; 106:2128-2137. [PubMed: 15914554]

14. Khorashad JS, Anand M, Marin D, et al. The presence of a BCR-ABL mutant allele in CML does not always explain clinical resistance to imatinib. Leukemia. 2006; 20:658-663. [PubMed: 16467863]

15. Baccarani M, Cortes J, Pane F, et al. Chronic myeloid leukemia: an update of concepts and management recommendations of European LeukemiaNet. J Clin Oncol. 2009; 27:6041-6051. [PubMed: 19884523]

16. NCCN. Clinical Practice Guidelines in Oncology: Chronic Myelogenous Leukemia. Version 2.2010. Available from: www.nccn.org/

17. Weisberg E, Griffin JD. Mechanism of resistance to the ABL tyrosine kinase inhibitor STI571 in BCR-ABL-transformed hematopoietic cell lines. Blood. 2000; 95:3498-3505. [PubMed: 10828035]

18. Mahon FX, Deininger MW, Schultheis B, et al. Selection and characterization of BCR-ABL positive cell lines with differential sensitivity to the tyrosine kinase inhibitor STI571; diverse mechanisms of resistance. Blood. 2000; 96:1070-1079. [PubMed: 10910924]

19. Le Coutre P, Tassi E, Varella-Garcia M, et al. Induction of resistance to the Abelson inhibitor STI571 in human leukemic cells through gene amplification. Blood. 2000; 95:1758-1766. [PubMed: 10688835]

20. Hochhaus A, La Rosee P. Imatinib therapy in chronic myelogenous leukemia: strategies to avoid and overcome resistance. Leukemia. 2004; 18:1321-1331. [PubMed: 15215876] 
21. Kantarjian HM, Larson RA, Guilhot F, et al. Efficacy of imatinib dose escalation in patients with chronic myeloid leukemia in chronic phase. Cancer. 2009; 115:551-560. [PubMed: 19117345]

22. Jabbour E, Kantarjian HM, Jones D, et al. Imatinib mesylate dose escalation is associated with durable responses in patients with chronic myeloid leukemia after cytogenetic failure on standarddose imatinib therapy. Blood. 2009; 113:2154-2560. [PubMed: 19060245]

23. Talpaz M, Silver RT, Druker BJ, et al. Imatinib induces durable hematology and cytogenetic responses in patients with accelerated phase chronic myeloid leukemia. Results of a phase 2 study. Blood. 2002; 99:1928-1937. [PubMed: 11877262]

24. Cortes JE, Kantarjian HM, Goldberg SL, et al. High-dose imatinib in newly diagnosed chronicphase chronic myeloid leukemia: high rates of rapid cytogenetic and molecular responses. J Clin Oncol. 2009; 27:4754-4759. [PubMed: 19720924]

25. Cortes J, Baccarani M, Guilhot F, et al. A phase III randomized, open-label study of daily imatinib mesylate $400 \mathrm{mg}$ versus $800 \mathrm{mg}$ in patients with newly diagnosed, previously untreated chronic myeloid leukemia in chronic phase using molecular endpoints: tyrosine kinase inhibitor optimization and selectivity study. J Clin Oncol. 2010; 28:424-430. [PubMed: 20008622]

26. Baccarani M, Druker BJ, Cortes-Franco J, et al. 24 months update of the TOPS study: a phase III, randomized, open-label study of $400 \mathrm{mg} / \mathrm{d}$ (SD-IM) versus $800 \mathrm{mg} / \mathrm{d}$ (HD-IM) of imatinib mesylate (IM) in patients (pts) with newly diagnosed, previously untreated chronic myeloid leukemia in chronic phase (CML-CP). Blood. 2009; 114(Suppl 1) Abstract 337.

27. Hehlmann R, Lauseker M, Jung-Munkqitz S, et al. Treatment optimization by high-dose imatinib: randomized comparison of imatinib $800 \mathrm{mg}$ versus imatinib $400 \mathrm{mg}+/-$ IFN in newly diagnosed BCR-ABL positive chronic phase (CP) CML: the German CML-study IV. J Clin Oncol. 2010; 28(Suppl) Abstract 6517.

28. Sprycel (dasatinib) [package insert]. Princeton, NJ: Bristol-Myers Squibb Co; Jun. 2009

29. Saglio G, Cortes JE, Schiffer CA, et al. Types of resistance to imatinib and other potential predictors of response to second-line dasatinib therapy. J Clin Oncol. 2010; 28(Suppl) Abstract 6569.

30. Frame D. New strategies in controlling drug resistance in chronic myeloid leukemia. Am J HealthSyst Pharm. 2007; 64:S16-S21.

31. Shah NP, Tran C, Lee FY, Chen P, Norris D, Sawyers CL. Overriding imatinib resistance with a novel ABL kinase inhibitor. Science. 2004; 305:399-401. [PubMed: 15256671]

32. O'Hare T, Walters DK, Stoffregen EP, et al. In vitro activity of Bcr-Abl inhibitors AMN107 and BMS-354825 against clinically relevant imatinib resistant Abl kinase domain mutants. Cancer Res. 2005; 65:4500-4505. [PubMed: 15930265]

33. Hochhaus A, Kantarjian HM, Baccarani M, et al. Dasatinib induces notable hematologic and cytogenetic responses in chronic-phase chronic myeloid leukemia after failure of imatinib therapy. Blood. 2007; 109:2303-2309. [PubMed: 17138817]

34. Hochhaus A, Baccarani M, Deininger M, et al. Dasatinib induces durable cytogenetic responses in patients with chronic myelogenous leukemia in chronic phase with resistance or intolerance to imatinib. Leukemia. 2002; 22:1200-1206.

35. Cervantes F, Baccarani M, Lipton J, et al. Dasatinib long-term efficacy in patients with chronic myeloid leukemia in chronic phase (CML-CP) with resistance or intolerance to imatinib: a twoyear update of the START-C study. Haematologica. 2008; 93(Suppl 1) Abstract 0934.

36. Kantarjian H, Pasquini R, Levy V, et al. Dasatinib or high-dose imatinib for chronic-phase chronic myeloid leukemia resistant to imatinib at a dose of 400 to $600 \mathrm{mg}$ daily: two-year follow-up of a randomized phase 2 study (START-R). Cancer. 2009; 115:4136-4147. [PubMed: 19536906]

37. Shah NP, Kantarjian HM, Kim DW, et al. Intermittent target inhibition with dasatinib $100 \mathrm{mg}$ once daily preserves efficacy and improves tolerability in imatinib-resistant and -intolerant chronicphase chronic myeloid leukemia. J Clin Oncol. 2008; 26:3204-3212. [PubMed: 18541900]

38. Shah NP, Kim S, Kantarjian H, et al. Potent, transient inhibition of BCR-ABL with dasatinib 100 $\mathrm{mg}$ daily achieve rapid and durable cytogenetic responses and high transformation-free survival rates in chronic phase chronic myeloid leukemia in patients with resistance, suboptimal response or intolerance to imatinib. Haematologica. 2010; 95:232-240. [PubMed: 20139391] 
39. Shah NP, Cortes JE, Schiffer CA, et al. Four-year follow-up of patients with chronic myeloid leukemia (CP-CML) receiving $100 \mathrm{mg}$ of dasatinib once daily. J Clin Oncol. 2010; 28(Suppl) Abstract 6512.

40. Kantarjian H, Cortes J, Kim DW, et al. Phase 3 study of dasatinib $140 \mathrm{mg}$ once daily versus $70 \mathrm{mg}$ twice daily in patients with chronic myeloid leukemia in accelerated phase resistant or intolerant to imatinib: 15-month median follow-up. Blood. 2009; 113:6322-6329. [PubMed: 19369231]

41. Kantarjian H, Shah NP, Hochhaus A, et al. Dasatinib versus imatinib in newly diagnosed chronicphase chronic myeloid leukemia. N Engl J Med. 2010; 362:2260-2270. [PubMed: 20525995]

42. Golemovic M, Verstovsek S, Giles F, et al. AMN107, a novel aminopyrimidine inhibitor of Bcr$\mathrm{Abl}$, has in vitro activity against imatinib-resistant chronic myeloid leukemia. Clin Cancer Res. 2005; 11:4941-4947. [PubMed: 16000593]

43. Weisberg E, Manley PW, Breitenstein W, et al. Characterization of AMN107, a selective inhibitor of native and mutant Bcr-Abl. Cancer Cell. 2005; 7:129-141. [PubMed: 15710326]

44. Hantschel O, Rix U, Superti-Furga G. Target spectrum of the BCR-ABL inhibitors imatinib, nilotinib, dasatinib. Leuk Lymphoma. 2008; 49:615-619. [PubMed: 18398720]

45. Tasigna (nilotinib) [package insert]. East Hanover, NJ: Novartis Pharmaceuticals Co; Aug. 2009

46. Kantarjian H, Giles F, Bhalla K, et al. Update on imatinib-resistant chronic myeloid leukemia patients in chronic phase (CML-CP) on nilotinib therapy at 24 months: clinical response, safety, and long-term outcomes. Blood. 2009; 114(Suppl 1) Abstract 1129.

47. Hochhaus A, Giles F, Apperely J, et al. Nilotinib in chronic myeloid leukemia patients in accelerated phase (CML-AP) with imatinib resistance or intolerance: 24 month follow-up results of a phase 2 study. Haematologica. 2009; 94(Suppl) Abstract 0631.

48. Cortes JE, Jones D, O'Brien S, et al. Nilotinib as front-line treatment for patients with chronic myeloid leukemia in early chronic phase. J Clin Oncol. 2010; 28:392-397. [PubMed: 20008621]

49. Rosti G, Castagnetti F, Palandri F, et al. Nilotinib $800 \mathrm{mg}$ daily as frontline therapy of Ph+chronic myeloid leukemia: dose delivered and safety profile for the GIMEMA CML working party. Blood. 2009; 114(Suppl 1) Abstract 2205.

50. O'Dwyer M, Kent E, Parker M, et al. Nilotinib $300 \mathrm{mg}$ twice daily is effective and well tolerated as first line treatment of Ph-positive chronic myeloid leukemia in chronic phase: preliminary results of the ICORG 0802 Phase 2 study. Blood. 2009; 114(Suppl 1) Abstract 3294.

51. Saglio G, Kim DW, Issaragrisil S, et al. Nilotinib versus imatinib for newly diagnosed chronic myeloid leukemia. N Engl J Med. 2010; 262:2251-2259.

52. Hino M, Matsumura I, Kurokawa M, et al. The effect of nilotinib on major molecular response (MMR) compared with imatinib in Japanese patients (pts) with newly diagnosed chronic myeloid leukemia in chronic phase (CML-CP): results from the Japanese subset of ENESTnd. J Clin Oncol. 2010; 28(Suppl) Abstract e17001.

53. Tasigna (nilotinib) [package insert]. East Hanover, NJ: Novartis; Jun. 2010

54. Gambacorti-Passerini C, Cortes JE, Khoury HJ, et al. Safety and efficacy of bosutinib in patients with $\mathrm{AP}$ and BP CML and Ph+ALL following resistance/intolerance to imatinib and other TKIs: update from study SKI-200. J Clin Oncol. 2010; 28(Suppl) Abstract 6509.

55. Cortes JE, Kantarjian H, Brummendorf T, et al. Safety and efficacy of bosutinib (SKI-606) in patients (pts) with chronic phase (CP) chronic myeloid leukemia (CML) following resistance or intolerance to imatinib (IM). J Clin Oncol. 2010; 28(Suppl) Abstract 6502.

56. Quintas-Cardama A, Kantarjian H, Cortes J. Homoharringtonine, omacetaxine mepesuccinate, and chronic myeloid leukemia circa 2009. Cancer. 2009; 115:5382-5393. [PubMed: 19739234]

57. Chen R, Gandhi V, Plunkett W. A sequential blockade strategy for the design of combination therapies to overcome oncogene addition in chronic myelogenous leukemia. Cancer Res. 2006; 66:10959-10966. [PubMed: 17108134]

58. Chen Y, Hu Y, Michaels S, Segal D, Brown D, Li S. Inhibitory effects of omacetaxine on leukemic stem cells and BCR-ABL induced chronic myeloid leukemia and acute lymphoblastic leukemia in mice. Leukemia. 2009; 23:1446-1454. [PubMed: 19322212]

59. Cortes-Franco J, Khoury HJ, Nicolini FE, et al. Safety and efficacy of subcutaneous-administered omacetaxine mepesuccinate in imatinib-resistant chronic myeloid leukemia (CML) patients who 
harbor the Bcr-Abl T315I mutation- results of an ongoing multicenter phase 2/3 study. Blood. 2009; 114(Suppl 1) Abstract 644.

60. Lipton JH, Wetzler M, Nicolini F, et al. Safety of omacetaxine mepesuccinate (OM) subcutaneous (SQ) injection for the treatment of chronic myeloid leukemia (CML) patients (pts) resistant or intolerant to tyrosine kinase inhibitors (TKIs): analysis of two phase II studies. J Clin Oncol. 2010; 28(Suppl) Abstract 6568.

61. Talpaz M, Cortes JE, Deininger MW, et al. Phase I trial of AP24534 in patients with refractory chronic myeloid leukemia (CML) and hematologic malignancies. J Clin Oncol. 2010; 28(Suppl) Abstract 6511.

62. Cortes-Franco J, Dombret H, Schafhausen P, et al. Danusertib hydrochloride (PHA-739358), a multi-kinase aurora inhibitor, elicits clinical benefit in advanced chronic myeloid leukemia and Philadelphia chromosome positive acute lymphoblastic leukemia. Blood. 2009; 114(Suppl 1) Abstract 864.

63. Van Etten RA, Chan WW, Zaleskas VM, et al. DCC-2036: A novel switch pocket inhibitor of ABL tyrosine kinase with therapeutic efficacy against BCR-ABL T315I in vitro and in a CML mouse model. Blood. 2007; 110(Suppl 1) Abstract 463.

64. Kurzrock R, Gutterman JU, Talpaz M. The molecular genetics of Philadelphia chromosomepositive leukemias. N Engl J Med. 1988; 319:990-998. [PubMed: 3047582]

65. Carpiuc KT, Stephens JM, Botteman MF, Feng W, Hay JW. A review of the clinical and economic outcomes of imatinib in Philadelphia chromosome-positive acute lymphoblastic leukemia. Expert Opin Pharmacother. 2007; 8:2775-2787. [PubMed: 17956198]

66. Druker BJ, Sawyers CL, Kantarjian H, et al. Activity of a specific inhibitor of the BCR-ABL tyrosine kinase in the blast crisis of chronic myeloid leukemia and acute lymphoblastic leukemia with the Philadelphia chromosome. N Engl J Med. 2001; 344:1038-1042. [PubMed: 11287973]

67. Ottmann OG, Druker BJ, Sawyers CL, et al. A phase 2 study of imatinib in patients with relapsed or refractory Philadelphia chromosome-positive acute lymphoid leukemias. Blood. 2002; 100:1965-1971. [PubMed: 12200353]

68. Ottmann OG, Wassmann B, Pfeifer H, et al. Imatinib compared with chemotherapy as front-line treatment of elderly patients with Philadelphia chromosome positive acute lymphoblastic leukemia (Ph+ALL). Cancer. 2007; 109:2068-2076. [PubMed: 17429836]

69. Vignetti M, Fazi P, Cimingo G, et al. Imatinib plus steroids induces complete remission and prolonged survival in elderly Philadelphia chromosome positive acute lymphoblastic leukemia without additional chemotherapy: results of the Grippo Italiano Malattie Ematologiche dell'Adulto (GIMEMA) LAL0201-protocol. Blood. 2007; 109:3676-3678. [PubMed: 17213285]

70. Thomas DA, Faderl S, Cortes J, et al. Treatment of Philadelphia chromosome-positive acute lymphocytic leukemia with hyper-CVAD and imatinib mesylate. Blood. 2004; 103:4396-4407. [PubMed: 14551133]

71. Thomas DA, O'Brien SM, Faderl S, et al. Long-term outcome after hyper-CVAD and imatinib (IM) for de novo or minimally treated Philadelphia-chromosome positive acute lymphoblastic leukemia (Ph-ALL). J Clin Oncol. 2010; 28(Suppl) Abstract 6506.

72. Towatari M, Yanada M, Usui N, et al. Combination of intensive chemotherapy and imatinib can rapidly induce high-quality complete remission for a majority of patients with newly diagnosed $B C R-A B L$-positive acute lymphoblastic leukemia. Blood. 2004; 104:3507-3512. [PubMed: 15315963]

73. Delannoy A, Delabesse E, Lheritier V, et al. Imatinib and methylprednisolone alternated with chemotherapy improve the outcome of elderly patients with Philadelphia-positive acute lymphoblastic leukemia: results of the GRAALL AFR09 study. Leukemia. 2006; 20:1526-1532. [PubMed: 16838024]

74. Wassmann B, Pfeifer H, Goekbuget N, et al. Alternating versus concurrent schedules of imatinib and chemotherapy as front-line therapy for Philadelphia-positive acute lymphoblastic leukemia ( $\mathrm{Ph}$ +ALL). Blood. 2006; 108:1469-1477. [PubMed: 16638934]

75. De Labarthe A, Rousselot P, Huguet-Rigal F, et al. Imatinib combined with induction or consolidation chemotherapy in patients with de novo Philadelphia chromosome-positive acute 
lymphoblastic leukemia: results of the GRAAPH-2003 study. Blood. 2007; 109:1408-1413. [PubMed: 17062730]

76. Chalandon Y, Thomas X, Hayette S, et al. First results of the GRAAPH-2005 study in younger adult patients with de novo Philadelphia positive acute lymphoblastic leukemia. Blood. 2008; 112(Suppl 1) Abstract 12.

77. Ottmann OG, Wassmann B. Imatinib in the treatment of Philadelphia chromosome-positive acute lymphoblastic leukemia: current status and evolving concepts. Best Pract Res Clin Haematol. $2003 ; 15: 757-769$.

78. Hoffmann WK, Komor M, Hoelzer D, Ottmann OG. Mechanisms of resistance to STI571 (imatinib) in Philadelphia-chromosome positive acute lymphoblastic leukemia. Leuk Lymphoma. 2004; 45:655-660. [PubMed: 15160936]

79. Jones D, Thomas D, Cameron Yin C, et al. Kinase domain point mutations in Philadelphia chromosome-positive acute lymphoblastic leukemia emerge alter therapy with BCR-ABL kinase inhibitors. Cancer. 2008; 113:985-994. [PubMed: 18615627]

80. Wassmann B, Scheuring U, Pfeifer H, et al. Efficacy and safety of imatinib mesylate (Glivec) in combination with interferon-a (IFN-a) in Philadelphia chromosome-positive acute lymphoblastic leukemia (Ph+ALL). Leukemia. 2003; 17:1919-1924. [PubMed: 14513038]

81. Cortes J, O'Brien SM, Pierce S, et al. The value of high-dose systemic chemotherapy and intrathecal chemotherapy for central nervous system prophylaxis in different risk groups of adult acute lymphoblastic leukemia. Blood. 1995; 86:2091-2097. [PubMed: 7662956]

82. Kantarjian HM, O'Brien S, Smith TL, et al. Results of treatment with hyper-CVAD, a doseintensive regimen, in adult acute lymphocytic leukemia. J Clin Oncol. 2000; 18:547-561. [PubMed: 10653870]

83. Petzer AL, Gunsilius E, Hayes M, et al. Low concentrations of STI571 in the cerebrospinal fluid: a case report. Br J Haematol. 2002; 117:623-625. [PubMed: 12028032]

84. Takayama N, Sato N, O’Brien SG, Ikeda Y, Okamoto S. Imatinib mesylate has limited activity against the central nervous system involvement of Philadelphia chromosome-positive acute lymphoblastic leukemia due to poor penetration into cerebrospinal fluid. Br J Haematol. 2002; 119:106-108. [PubMed: 12358909]

85. Ottmann O, Dombret H, Martinelli G, et al. Dasatinib induces rapid hematologic and cytogenetic responses in adult patients with Philadelphia chromosome-positive acute lymphoblastic leukemia with resistance or intolerance to imatinib: interim results of a phase 2 study. Blood. 2007; 110:2309-2315. [PubMed: 17496201]

86. Foa R, Vitale A, Guarini A, et al. Line treatment of adult Ph+acute lymphoblastic leukemia (ALL) patients. Final results of the GIMEMA LAL1205 study. Blood. 2008; 112(Suppl 1) Abstract 305.

87. Lilly MB, Ottmann OG, Shah NP, et al. Dasatinib $140 \mathrm{mg}$ once daily versus $70 \mathrm{mg}$ twice daily in patients with $\mathrm{Ph}$-positive acute lymphoblastic leukemia who failed imatinib: results from a phase 3 study. Am J Hematol. 2010; 85:164-170. [PubMed: 20131302]

88. Rousselot P, Cayuela JM, Recher C, et al. Dasatinib (Sprycel) and chemotherapy for first-line treatment in elderly patients with de novo Philadelphia positive ALL: results of the first 22 patients included in the EWALL-Ph-01 trial (on behalf of the European Working Group on Adult ALL (EWALL)). Blood. 2008; 112(Suppl 1) Abstract 2920.

89. Ravandi F, Kantarjian H, Thomas DA, et al. Phase II study of combination of the hypercvad regimen with dasatinib in the front line therapy of patients with Philadelphia chromosome $(\mathrm{Ph})$ positive acute lymphoblastic leukemia (ALL). Blood. 2009; 114(Suppl 1) Abstract 837.

90. Ravandi F, Kantarjian H, Cortes J, et al. Combinations of the hypercvad regimen with dasatinib is effective in patients with relapsed Philadelphia chromosome $(\mathrm{Ph})$ positive acute lymphoblastic leukemia and lymphoid blast phase chronic myeloid leukemia (CML-LB). Blood. 2009; 114(Suppl 1) Abstract 2043. 


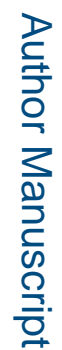

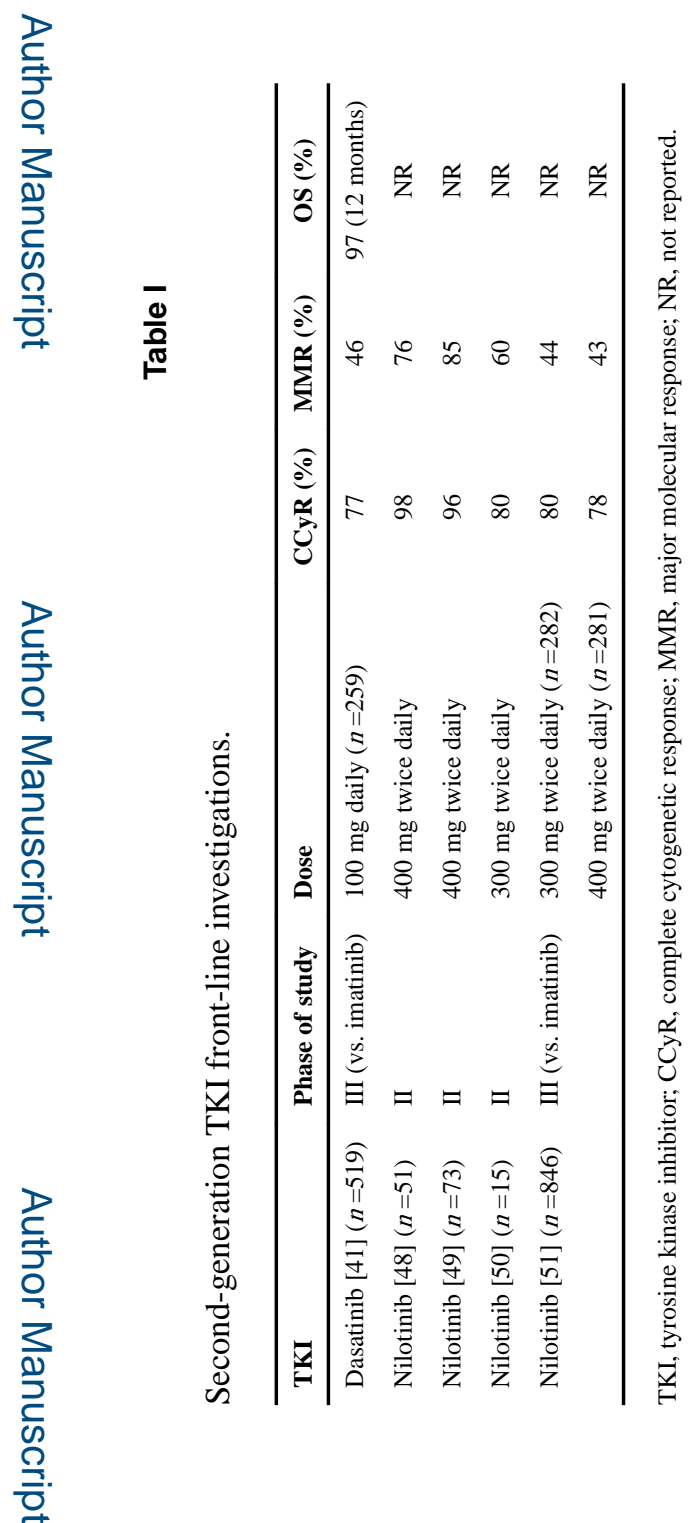

Leuk Lymphoma. Author manuscript; available in PMC 2017 November 10. 
Table II

Imatinib-based combination chemotherapy in Ph-positive ALL.

\begin{tabular}{llcrc}
\hline Investigator & Regimen & Number of patients & CR (\%) & OS (\%) \\
\hline MDACC [71] & Hyper-CVAD & 54 & 93 & 54 (36 months) \\
JALSG [72] & ALL202 & 24 & 96 & 89 (12 months) \\
GRAALL [73] & AFR09 & 30 & 90 & 66 (12 months) \\
GRAALL [75] & GRAAPH-2003 & 45 & 96 & 65 (18 months) \\
GRAALL [76] & Vincristine + dexamethasone & 42 & 100 & 68 (24 months) \\
& Hyper-CVAD & 41 & 95 & 54 (24 months) \\
\hline
\end{tabular}

Ph-positive ALL, Philadelphia-positive acute lymphoblastic leukemia; MDACC, M. D. Anderson Cancer Center; JALSG, Japan Adult Leukemia Study Group; GRAALL, Group for Research on Adult Acute Lymphoblastic Leukemia; hyper-CVAD, hyper-fractionated cyclophosphamide, vincristine, doxorubicin, and dexamethasone; CR, complete remission; OS, overall survival. 